\title{
Study on the fractal gradation optimization of ATB-25
}

\author{
Dong Weizhi, a , Guo Xuedong ${ }^{2, b}$ and Gu Liang ${ }^{3, c}$ \\ 1,2 College of Traffic, Ji Lin University, Changchun, China \\ 1,3 Jilin Institute of Architecture and Civil Engineering, Changchun, China \\ a dongweizhi1022@126.com, b guoxd@jlu.edu.cn, c xiaozhi0723@163.com
}

Keywords: ATB; gradation optimization; fractal theory; fractal dimension; correlation coefficient

\begin{abstract}
Many diseases of the high grade highway asphalt pavement in our country such as lack of durability and being distressed early have close relationships with improper gradation design. In this paper, based on the fractal theory, fractal dimension range was obtained through Marshall Test results of type ATB-25 as the result of the fractal dimension analysis and Marshall Test parameters. And target gradation was recommended according to the different gradation curve of fractal dimension and the degree of fitting. The results show that the optimization design method of asphalt mixture can meet the standard requirements.
\end{abstract}

\section{Introduction}

Fractal theory is new subject to describe quantitatively the geometric complexity and space filling capability of the object [1]. The method is based on fractal geometry of the object and the self similarity experiment. Asphalt mixture consist of mineral aggregates and asphalt which are mixed [2], the material aggregates are the main component of asphalt mixture and the proportion can reach about 95\% which leads to that the stability and strength of the mixture depends on the species, specifications, shape, surface structure and grading of them. Because Mineral aggregate gradation Structure the formed with the vital role, therefore the properties of asphalt mixture in the very great $d$ has very strong self similarity, the fractal theory in the gradation design optimization studies will play an important role.

\section{Fractal Dimension and Fractal}

Fractal theory is a new theory. The concept of fractal was first introduced by the American mathematician Mandelbrot (B. B. Mandelbrot). And characteristic of the partial and whole self-similar the theory was first mentioned in the book of "How long is the British coast line?" .Fractal theory includes e two principles, one is the principle of similarity, the other is iterative generation principle [3]. That shows that the fractal invariant will be the same under the usual geometric transform (scaling). In the study of asphalt mixture, fractal dimension is used to describe the characteristics of gradation, and we use the factor to the gradation design to set up the relationship between the factor and performance of asphalt pavement. On this basis, we can use the fractal dimension variation to optimize the gradation of asphalt mixture.

\section{Asphalt Mixture Fractal Theory Research}

By the study of the fractal theory of the rock fragmentation, fractal theory applies to aggregate although the s physical process is very complicated, random and irregular [4].

The present gradation is all base on the passing rate. If the mass distribution of the aggregate is studied with the theory of fractal dimension, then we can get the following function

$$
P(\mathrm{r})=\frac{\mathrm{r}^{3-\mathrm{D}}-\mathrm{r}_{\min }^{3-\mathrm{D}}}{\mathrm{r}_{\max }^{3-\mathrm{D}}-\mathrm{r}_{\min }^{3-\mathrm{D}}}
$$

where: $P(\mathrm{r})$ - the passing rate of aggregater 
$r_{\max }, r_{\min }$ - the maximum particle size, the minimum particle size

Different grading curve can be obtained according to the given $\mathrm{D}$ value, and the variation of $\mathrm{D}$ value determines the types of gradation curve. If the $r_{\min }$ can be neglected because its little value, then formula (2):

$P(r)=\left(\frac{\mathrm{r}}{\mathrm{r}_{\max }}\right)^{3-\mathrm{D}}$

If $M$ was the total quality, $\rho$ is the aggregate density, the volume is:

$V=\frac{M}{\rho}$

With the formula (2) and (3), we can get formula (4):

$P(\mathrm{r})=\frac{M_{\mathrm{r}}}{M}=\frac{V_{\mathrm{r}}}{V}=\left(\frac{\mathrm{r}}{\mathrm{r}_{\max }}\right)^{3-\mathrm{D}}$

where: $M r$ - fractal total quality of aggregates which particle sizes are less than $\mathrm{R}$ particle

$V$-fractal total volume of aggregates which particle sizes are less than R particle

The analysis: Mass distribution of the particle can be converted to the fractal dimension $\mathrm{D}$ and is the same as the volume distribution. The passing rate of different particle can also be denoted by fractal dimension. According to fractal dimension, the variation range of the fractal dimension of the aggregate the topological dimension and the dimension of the space, that is, $2<\mathrm{D}<3$ [5]. At present, the main method used to calculate the gradation fractal dimension is to calculate slope method according to the double logarithmic coordinates of $\ln (M r / M)$ and $\ln r$ using linear regression equation. In this process, the fractal dimension can be worked out using the slope of the double logarithmic coordinates $\ln (M r / M)$ and $\ln r$, and the correlation coefficient can also be used as the basic parameter, as the following:

$$
\ln \frac{M_{\mathrm{r}}}{M}=(3-\mathrm{D}) \ln \mathrm{r}+\mathrm{a}
$$

According to the formula of $\mathrm{D}=3-\mathrm{b}$.

Mineral aggregate gradation will change with the variation of the fractal dimension change, so to set up the relationship between the new gradations with the traditional grading formula is t necessary [6]. The parameters of traditional calculation methods can convert each other. By contraction, the relationship of fractal theory formula and traditional calculating methods can be obtained. If the parameter in formula (2) was replaced by that in the 3-D, we can get the same function, that is: $\mathrm{D}=3-\mathrm{n}$. Through the study, fractal theory gradation calculation method can not only express the traditional grading method, but also can reflect the gradation characteristics, and this will optimize the mixture design to a large extend.

\section{Experiment Study on Asphalt Mixture Fractal}

Experimental results show, the median value in the specifications often does not make the mixture densely enough. The purpose of our study is to find a simple, accurate method to optimize the mixture design. According to Ji-Cao expressway, we can get the following 11 gradation curve and we will study fractal dimension with the methods of orthogonal design optimization and composition of mineral materials design. The curves are shown in figure 1.

Then, we compute the logarithm of the data of the 11 ATB-25 grading curve and the sieve aperture, and a linear regression equation can be got using the least square method. On this basis, correlation coefficient $R^{2}$ and fractal dimension $\mathrm{D}$ can be worked out. The results are shown in table 1 .

Table 1 Fractal dimension and correlation coefficients of ATB-25 gradation

\begin{tabular}{|c|c|c|}
\hline Grade number & Fractal dimension D & Correlation coefficient $R^{2}$ \\
\hline Number 1 & 2.5044 & 0.9704 \\
\hline Number 2 & 2.5428 & 0.9620 \\
\hline
\end{tabular}




\begin{tabular}{|c|c|c|}
\hline Numbe 3 & 2.4475 & 0.9777 \\
\hline Number 4 & 2.4966 & 0.9729 \\
\hline Number 5 & 2.5338 & 0.9678 \\
\hline Number 6 & 2.4409 & 0.9838 \\
\hline Number 7 & 2.5130 & 0.9648 \\
\hline Number 8 & 2.5616 & 0.9511 \\
\hline Number 9 & 2.4548 & 0.9745 \\
\hline Number 10 & 2.4314 & 0.9694 \\
\hline Number 11 & 2.5534 & 0.9698 \\
\hline
\end{tabular}

The data of1-11\# grading showed us that they correlated and fitted very well each other. We can also be clear of aggregate gradation from the fractal dimension.

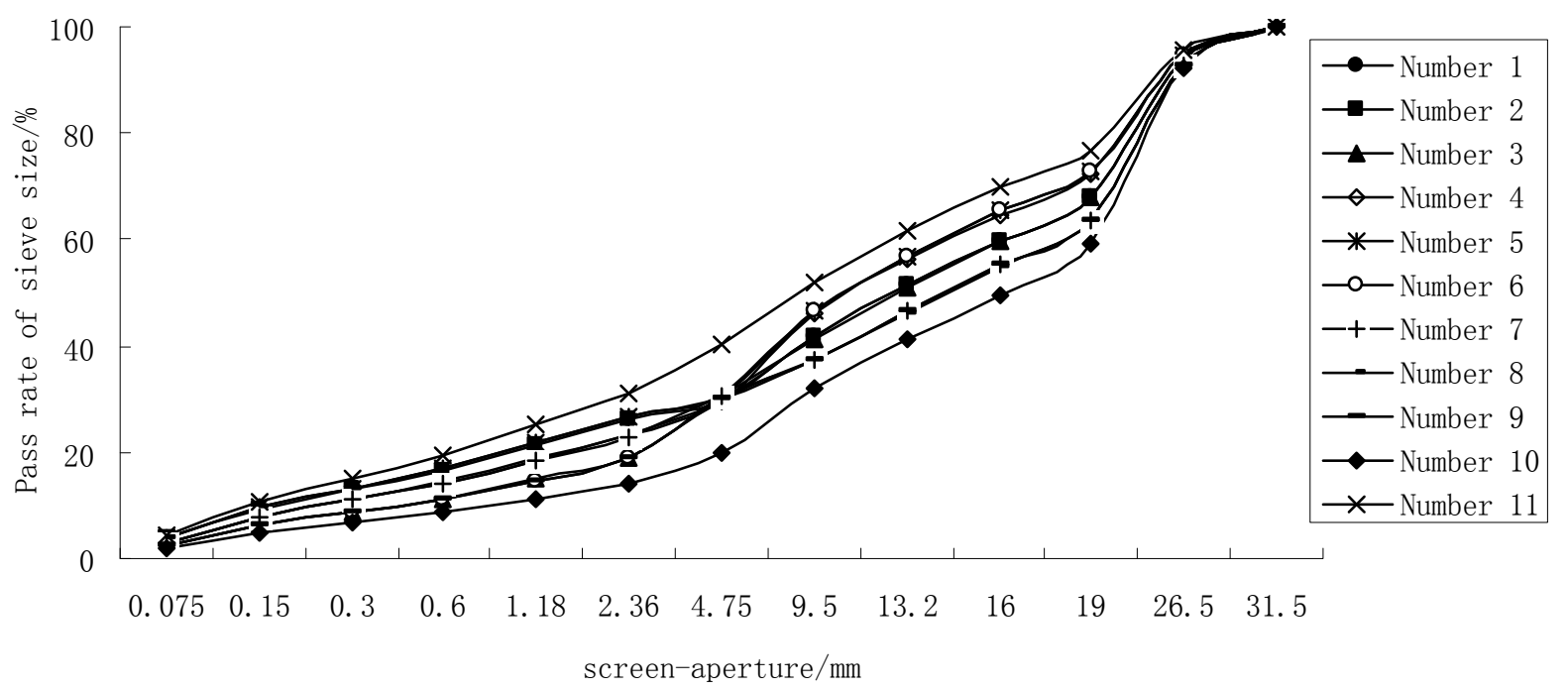

Fig. 1 ATB-25 Synthesis grading curve

\section{Analysis of Fractal dimension and road performance}

To carry out Marshall Test, 4\% the optimum asphalt content was selected of ATB-25. From the results of tests of 1-11\# in Fig. 1, we can get the following relationship between performance index and the fractal dimension, as shown in figure 2.
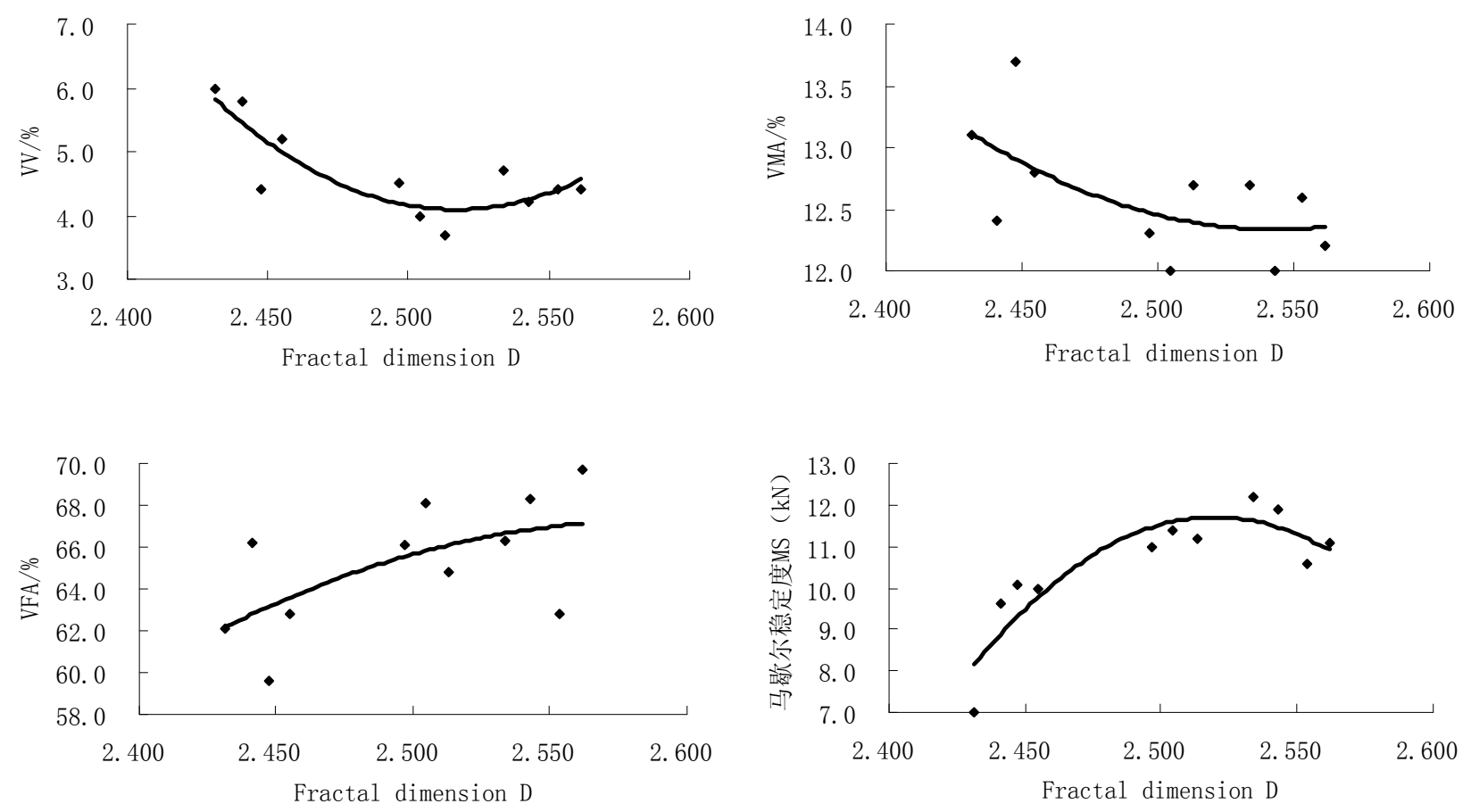

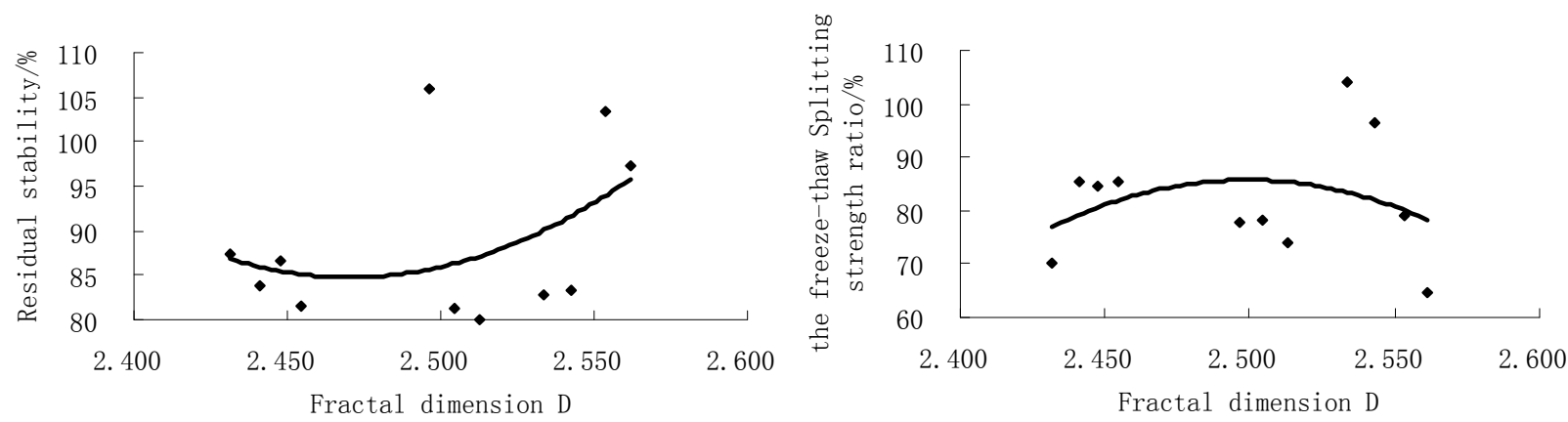

Fig.2 ATB-25 road performance index and fractal dimension diagram

Figure 2 shows that void ratio and voids in mineral aggregate increases with fractal dimension D which indicates that with the increase of gradation, the fractal dimension increases, the aggregate space filling ability is more strong, so the mixture void ratio is smaller, VMA also reduced. According to the related literatures, when the asphalt mixture void ratio is greater than $6 \%$,the pavement will leakage, and when it is less than $3 \%$, the pavement will cause bleed. To select the target porosity of $4 \%$ is the most appropriate. The fractal dimension of $4 \%$ is 2.5044 , and the fractal dimension curve tends to vary smoothly.

With the increase of fractal dimension of aggregate, fine aggregate increases gradually, which makes the grading continuous tapering, surface area increase, specific mixture density increase, and porosity decrease. These excess fine material and bitumen will form bitumen mortar which leads to the asphalt area increased, the cohesive force by asphalt coated aggregate particles increased, and the strength of asphalt mixture increased. With the increase of the amount of fine mineral aggregate and the reduction of the amount of coarse aggregate, the skeleton structure cannot be easily formed. This will influence the Marshall stability of mixture. And the curve of Marshall Stability will form a hump curve. The larger values of Marshall Stability appear between2.49and 2.55(the fractal dimension), and the peak point is 2.5197 . The value will decrease gradually after this peak point. The relationship of between the Water stability of the mixture and the fractal dimension is almost the same as that of Marshall Stability. Asphalt mixture has the smallest void ratio, maximum density, a high Marshall stability when the value is 2.51 . So it is enough of the mixture to resist water damage.

\section{The optimal gradation design}

From the characteristics of gradation fractal, we know that the parameter D can reflect the mineral aggregate gradation composition characteristics, and the correlation coefficient $\mathrm{R}^{2}$ can reflect the mineral aggregate gradation continuity. The greater $R^{2}$ is, the more the gradation is [7]. In this case, the average value of $R^{2}$ was 0.9695 , and we can see that the $1 \#, 3 \#, 4 \#, 6 \#, 9 \#, 11 \#$ gradation are more continuous and homogenous better than others. From the grading curve of ATB-25 in table 1, we can easy conclude that among the six gradation curves, the $\mathrm{D}$ values of $1 \#$ and $4 \#$ are between 2.49 and 2.55. Their Marshall Stability and water stability are satisfactory. So we select $1 \#$ and $4 \#$ as the optimal curve, as in table 2.

Table 2 Grading curve

\begin{tabular}{|c|c|c|c|c|c|c|c|c|c|c|c|c|c|}
\hline Grade & \multicolumn{10}{|c|}{ Pass rate of sieve size/\% } \\
\cline { 2 - 27 } & 31.5 & 26.5 & 19 & 16 & 13.2 & 9.5 & 4.75 & 2.36 & 1.18 & 0.6 & 0.3 & 0.15 & 0.075 \\
\hline Number 1 & 100 & 93.7 & 67.9 & 59.8 & 51.3 & 41.7 & 29.8 & 23.1 & 18.6 & 14.2 & 11.1 & 7.9 & 3.0 \\
\hline Number 4 & 100 & 94.6 & 72.2 & 64.8 & 56.2 & 45.9 & 29.5 & 23.4 & 18.9 & 14.5 & 11.3 & 8.0 & 3.0 \\
\hline
\end{tabular}

We adjusted the values of $1 \#$ and $4 \#$ in table 2 and recommended the gradation in Table 3 as the target one.

Table 3 Recommended target gradation

\begin{tabular}{|c|c|c|c|c|c|c|c|c|c|c|c|c|c|}
\hline Sieve size & 31.5 & 26.5 & 19.0 & 16.0 & 13.2 & 9.5 & 4.75 & 2.36 & 1.18 & 0.6 & 0.3 & 0.15 & 0.075 \\
\hline $\begin{array}{c}\text { Target } \\
\text { mixture ratio }\end{array}$ & 100 & 94.15 & 70.05 & 62.3 & 53.75 & 43.8 & 29.65 & 23.25 & 18.75 & 14.35 & 11.2 & 7.95 & 3.0 \\
\hline
\end{tabular}


We did the experiments to test the Pavement performance and the results showed that the above optimization method for asphalt mixture design can fully o meet the requirements of the standard.

\section{Acknowledgment}

This research was supported by the projects in science and technology research of department of education of Jilin province during the Eleventh Five-Year Plan Period, China. (Grant No. 2009177).

\section{References}

[1] Long Qiyi, Long Qiwei and Mu Zaile. Ideal fractal model and the actual nested structure. Physics, Vol. 23 (1994), p. 119-164

[2] Technical Specification for Construction of Highway Asphalt Pavements(JTG F40-2004). Beijing: China Communications Press (2004)

[3] Tang Ming, Ba Hengjing. Evaluation of topologic and fractal characteristics of concrete. Journal of Harbin University of Civil Engineering and Architecture, Vol. 35 (2002), p. 86-89

[4] Chen Guomin, Tanyiqiu and Wang Zheren. Fractal Study of Grading Curve Trend of the Asphalt Mixtures. Journal of Highway and Transportation Research and Development, Vol. 22 (2002), p. 1-4 [5] Jiang Shuangquan, Zhang Zhengqi and Yang Bo. Application of Fractal Geometry Theory to Study on Asphalt Mixture. Highway, 10 (2009), p. 198-203

[6] Chu Wuyang. Materials Science in fractal. Beijing: Chemical Industry Press (2004)

[7] Wang Yanli. Study on Asphalt Mixture Gradation Optimization.Chang'an University (2008) 